\title{
Object Tracking Based on Multi-feature Mean-shift Algorithm
}

\author{
Jiafu Jiang \\ Department of Computer \& Communication Engineering \\ Changsha University of Science \& Technology \\ Changsha, China \\ jjf_cscu@163.com
}

\author{
Hui Xiong \\ Department of Computer \& Communication Engineering \\ Changsha University of Science \& Technology \\ Changsha, China \\ xionghui_happy@163.com
}

\begin{abstract}
Object tracking is one of the key technologies in intelligent video surveillance and how to describe the moving target is a key issue. Traditional color histogram Mean-shift (MS) algorithm only considered object's color statistical information, and didn't contain object's space information, so when the object color was close to the background color, the traditional MS algorithm easily caused object tracking inaccurately or lost. To solve this problem, a novel object tracking algorithm which based on improved MS and Kalman was proposed in this paper. Firstly, using the joint color-texture histogram to represent a target and applying it to the MS framework, then the improved MS goes for a small range of search and target match according to the result of Kalman prediction. The experimental results show that our algorithm tracking the object accurately and effectively even though the object color was close to the background color or the target moves fast.
\end{abstract}

Keywords-object trackingn; Mean-shift; color-texture
histogram;Kalman

\section{INTRODUCTION}

Real-time object tracking is a critical task in the field of computer vision. It has been widely used in electronic monitoring system, intelligent traffic management, medical, military, etc [1]. And object tracking remains a challenge due to the illumination changes, object deformation, postural change or the target moves fast. Many tracking algorithms are based on the surface characteristics of the object, namely, in each frame to find the most similar region with the object surface model.

Recently, a lot of tracking algorithm emerged, such as Mean-shift algorithm [2], Kalman filter algorithm [3], particle filter algorithm, etc. the MS algorithm has gained significant attention as an efficient and robust method for visual tracking. As the traditional color histogram MS algorithm caused object tracking inaccurately or lost easily when the object color was close to the background color or the target moves fast. To solve these problems, many researchers proposed various improved methods in recent years, For example, to modeling characteristics of the object better, Yuan et al [4] use the color and texture as the target feature, and then use the detected object contour to track the target; Li et al [5] proposed an object tracking algorithm based on color texture histogram with the right to block average drift. However, MS could not adapt to it when the object moves fast. Kalman filter is a typical tracking algorithm, using the Kalman filter can achieve the estimation of the location of the fast object. [6] predict the results of the Kalman Filter and MS calculation results weighted to determine the final target location; [7] using the prediction of the Kalman filter as the initial search center in the MS algorithm and achieve a good result in the case of sudden change in target speed or the object moves fast.

To sum up, the main problem is that the feature of MS is signal, easily lead to track failure in the light mutation or the object color was close to the background color. And when the object moves fast or the speed changes suddenly, the MS could not predict accurately the object location in the next frame.

In this paper, two steps to achieve object tracking. The first step is adopt the LBP scheme to represent the target texture feature and then propose a joint color-texture histogram method for a more distinctive and effective target representation and applying it to the MS framework. The second step is the improved MS goes for a small range of search and target match according to the result of Kalman prediction. Experimental results show that the proposed algorithm can be a good deal with object color close to background color as well as tracking object moves too fast. In the rest of this paper, we will give a detailed descriptor of our algorithm and some contrast results.

\section{THE MULTI-FEATURE MS ALGORITHM}

\section{A. $\quad L B P$}

The texture of object is a relatively stable feature that could reflect the information of the object space characteristics, usually not subject to the impact of light and background color. The LBP [7] operator labels the pixel in an image by threshold its neighborhood with the center value and considering the result as a binary number (binary pattern). The general version of the LBP operator is defined as follows:

$$
L B P_{P, R}\left(x_{c}, y_{c}\right)=\sum_{p=0}^{P-1} s\left(g_{p}-g_{c}\right) 2^{p}
$$

Where $g_{c}$ corresponds to the gray value of the center pixel $\left(x_{c}, y_{c}\right)$ of a local neighborhood and $g_{p}$ to the gray values of $\mathrm{P}$ equally spaced pixels on a circle with radius $\mathrm{R}$. The function $\mathrm{s}(\mathrm{x})$ is defined as follows: 


$$
s(x)=\left\{\begin{array}{l}
1, x \geq 0 \\
0, x<0
\end{array}\right.
$$

Figure. 1 is an example of LBP $(\mathrm{P}=8, \mathrm{R}=1)$. Compared the eight gray value with the center. If it is greater than the center gray value, then represented by 1 , Otherwise, represented by 0 .

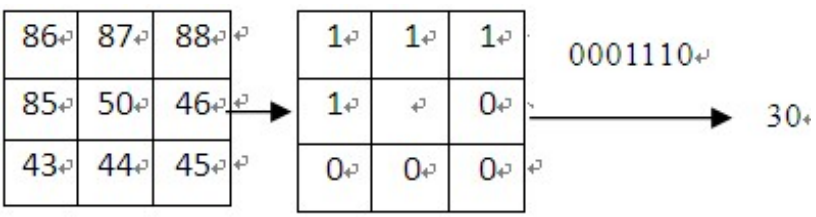

Figure1. An example of LBP8 texture model.

However, the LBP operator, obtained by the formula (1) does not have the rotation invariant. For this deficiency, [13] gives an extension of the LBP uniform pattern, defined as follows:

$$
L B P_{P, R}^{r i}=\min \left(R O R\left(L B P_{P, R}^{r i}, i\right) \mid i=0,1, \ldots, P-1\right)
$$

$$
L B P_{P, R}^{r i} \text { represent the LBP uniform pattern, ROR (x, i ) }
$$

on behalf of the P-bit binary number bit right shift of the $i$. Formula can be seen, for those who continue to rotate the LBP model taken the minimum the LBP rotation invariant pattern. In this paper, we adopt [8] to calculate the object texture feature.

\section{B. MS algorithm}

MS is a semi-automatic tracking method and it need to manually select the object in the initial frame. Denote by $X_{i}$ the normalized pixel positions in the target region, which is supposed to be centered at the origin point. The object model is computed as:

$$
q_{u}^{c}=c \sum_{i=1}^{n} k\left(\left\|\frac{x_{i}-x_{0}}{h}\right\|^{2}\right) \delta\left[b\left(x_{i}-u\right)\right]
$$

Where $\mathrm{u}$ represents the eigenvalue of the target model, $b\left(x_{i}\right)$ associates the pixel $x_{i}$ to the histogram bin, $\mathrm{k}(\mathrm{x})$ is an isotropic kernel function and constant $\mathrm{c}$ is standardized coefficient function $\sum_{u=1}^{m} q_{u}^{c}=1$.

Similarly, the target candidate model corresponding to the candidate region is given by

$$
p_{u}^{c}(y)=c \sum_{i=1}^{n} k\left(\left\|\frac{x_{i}^{*}-y_{0}}{h}\right\|^{2}\right) \delta\left[b\left(x_{i}^{*}\right)-u\right]
$$

Based on the Bhattacharyya, a metric which aims to estimate the similarity between the target model and the candidate model is defined.

$$
\rho(y)=\rho[p(y), q]=\sum_{u=1}^{m} \sqrt{p_{u}^{c}(y) q_{u}^{c}}
$$

More similar between the two density distribution, $\rho$ is greater. Find the maximum of the density estimation to obtain the new location of $y$ by using MS vector.

Where

$$
y=\frac{\sum_{i=1}^{n} y_{i} w_{i} g\left[\left\|\frac{\left(y_{0}-y_{i}\right)}{h}\right\|^{2}\right]}{\sum_{i=1}^{n} w_{i} g\left[\left\|\frac{\left(y_{0}-y_{i}\right)}{h}\right\|^{2}\right]}
$$

$$
w_{i}=\sum_{i=1}^{m} \delta\left[b\left(x_{i}\right)-u\right] \sqrt{\frac{q_{u}}{p_{u}\left(y_{0}\right)}} g(x)=-k^{\prime}(x) .
$$

\section{The MS algorithm with the joint color-texture histogram}

Each pixel is described with LBP texture-color features for the tracking area. Using the LBP uniform pattern, so there are $\mathrm{P}+2$ kinds of texture. We will select the five kinds of texture mode references to [9]. Comprehensive three-dimensional RGB color channels and the one-dimensional texture channel can define the joint color-texture histogram, so the probability density of the object feature of (4) is rewritten as:

$$
\left\{\begin{array}{l}
q=\left\{q_{u}^{c}\right\}_{u=1, \ldots, m} \\
q_{u}=C \sum_{i=1}^{N} k\left(\left\|x_{i}^{*}\right\|^{2}\right) \delta\left[\operatorname{bin}\left(x_{i}\right)-u\right]
\end{array}\right.
$$

Where $\operatorname{bin}\left(x_{i}\right)$ means the pixel $x_{i}$ to the color-texture histogram bin, $\mathrm{C}$ is normalization constants defined by

$$
C=\frac{1}{\sum_{i=1}^{n} k\left(\left\|x_{i}^{*}\right\|^{2}\right)}
$$

In the experiment, $m=8 \times 8 \times 8 \times 5$, The three dimensions represent the quantized bins of color channels and the fourth dimension is the bin of the $L B P_{P, R}^{r i}$ texture patterns.

\section{OBJECT TRACKING}

\section{A. The Kalman Filter}

The MS algorithm through its convergence, the iterative calculation of the MS vector in the current frame, and eventually converge to the true location of the target to achieve the purpose of tracking. So the MS tracking algorithm requires a certain degree of overlap region between the target template and the candidate object in the two adjacent frames. The MS could not track accurately when the object moves fast or the search range is small. So, in this paper, we use Kalman filter to solve this problem.

Kalman filter has been widely used in the state estimation and tracking field as an effective means. Kalman filter is an algorithm that estimates the linear minimum variance of the sequence of states of the dynamic system. Describe a dynamic system by using the state equation and observation equation. 
And it can begin testing as a starting point from any point method using recursive filtering. With small amount of calculation, the characteristics of the real-time calculation.

The state equation and the observation equation as follows:

$$
\begin{aligned}
& x(\mathrm{t})=\mathrm{Ax}(\mathrm{t}-1)+\mathrm{w}(\mathrm{t}-1) \\
& z(\mathrm{t})=\mathrm{Hx}(\mathrm{t})+\mathrm{v}(\mathrm{t})
\end{aligned}
$$

Where A denotes the state transition matrix and $\mathrm{H}$ denotes the measurement matrix. And $\mathrm{w}(\mathrm{t}-1)$ means the process noise and $\mathrm{v}(\mathrm{t})$ means the measurement noise, assuming that they are independent and follow a normal distribution Gaussian white noise. In this paper, we only predict the object position. Define Kalman filter system status $\mathrm{x}$ as a four-dimensional vector $\left(x_{t}, y_{t}, v x_{t}, v y_{t}\right)$, and $x_{t}, y_{t}, v x_{t}, v y_{t}$ are the target position and velocity in the $\mathrm{x}$-axis and $\mathrm{y}$-axis direction. Suppose the object do uniform motion in a unit time interval, so the state transition matrix $\mathrm{A}$ and the measurement matrix $\mathrm{H}$ can be defined ( $\Delta t$ is the time difference between two frames):

$$
\mathrm{A}=\left[\begin{array}{cccc}
1 & 0 & \Delta t & 0 \\
0 & 1 & 0 & \Delta t \\
0 & 0 & 1 & 0 \\
0 & 0 & 0 & 1
\end{array}\right]
$$

$$
H=\left[\begin{array}{llll}
1 & 0 & 0 & 0 \\
0 & 1 & 0 & 0
\end{array}\right]
$$

The position of the moving object predicted by the Kalman filter estimation will as the initial iteration of the MS algorithm. And then MS find the best match in their neighborhood. It can reduce the amount of computation and be able to adapt to the rapid move by using the Kalman filter.

\section{B. The tracking algorithm with the proposed MS and Kalman}

The traditional color-based MS algorithm for object tracking only concern the similarity of the candidate goals and the object template on the color distribution, without taking into account the information of the object movement direction in real space and velocity, etc., And when the object moves too fast, the MS algorithm can not accurately predict the location of the object in the next frame, resulting in tracking failure.

In this paper, based on color-texture features of MS tracking algorithm and joined the movement on the object position that Kalman filter predict at the same time. Get the center of object in each frame as the initial iteration of MS and it will find the optimal location of the object in the neighborhood within the scope of this. So the result will be the observations as the Kalman filter to compute in the next frame. Figure. 2 is the flow chart of tracking algorithm for this article. The algorithm steps are as follows:

1) Select region of interest in the initial frame, determine the tracking object. Calculate the joint color-texture histogram.

2) Initialize the object state vector of the Kalman filter and read the next frame, using (9), (10) to predict the center of the object location.

3) Step 2 results as the iterative initial value of the MS algorithm, using (7) to calculate the optimal location of the candidate object.
4) Using (6) to calculate the similarity between the object template and the candidate region. The step 3 result will as the observation of Kalman filter in the next frame.

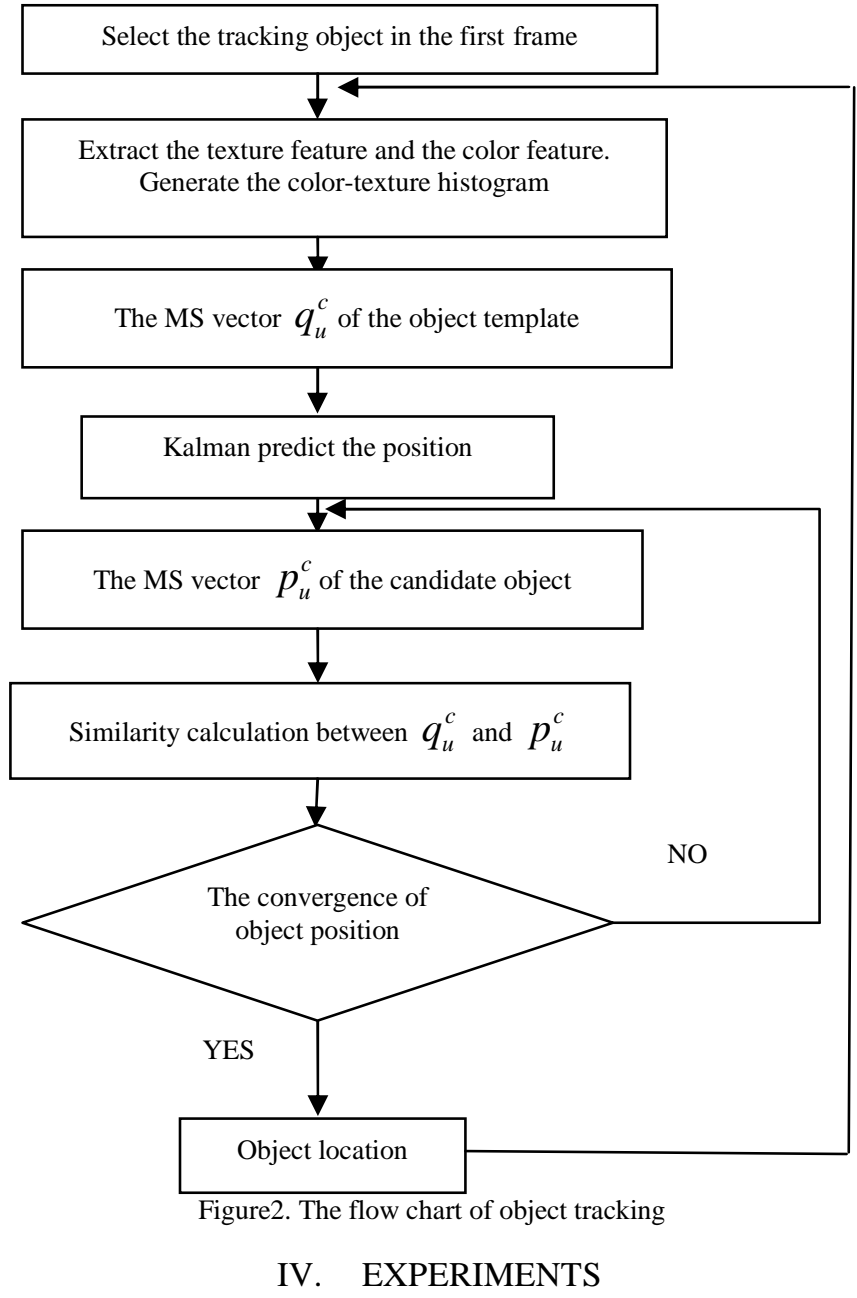

In our experiments, we select some standard test video sequences in order to verify the validity of our algorithm.

Experiment one proved the superiority compared to the color-texture histogram features and color histogram features. Select a table tennis video sequence to track the athlete's head. The human face color is similar to the background color in the video sequence. So the tracking results may not be good if using the color histogram features. In this section, experiments are performed to illustrate and testify the proposed joint colortexture model based MS tracking algorithm in comparison with the original MS. Figure. 3 shows the results and the black rectangle for tracking results based on color histogram features, the red for this article. It can be seen that the proposed algorithm has better robustness when the object color is close to the background region. 


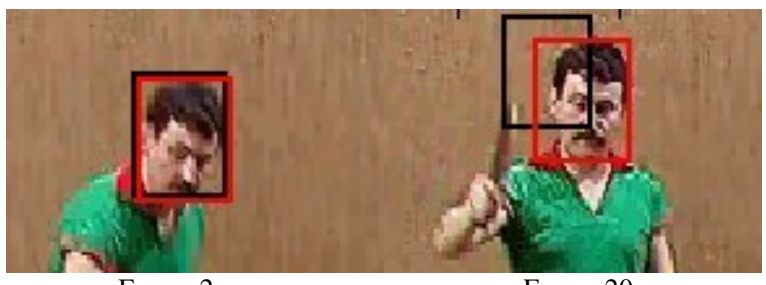

Frames2 Frames20

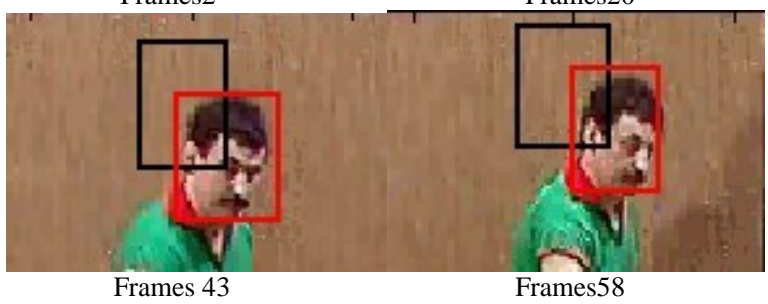

Figure3. The comparison between the proposed MS and the original
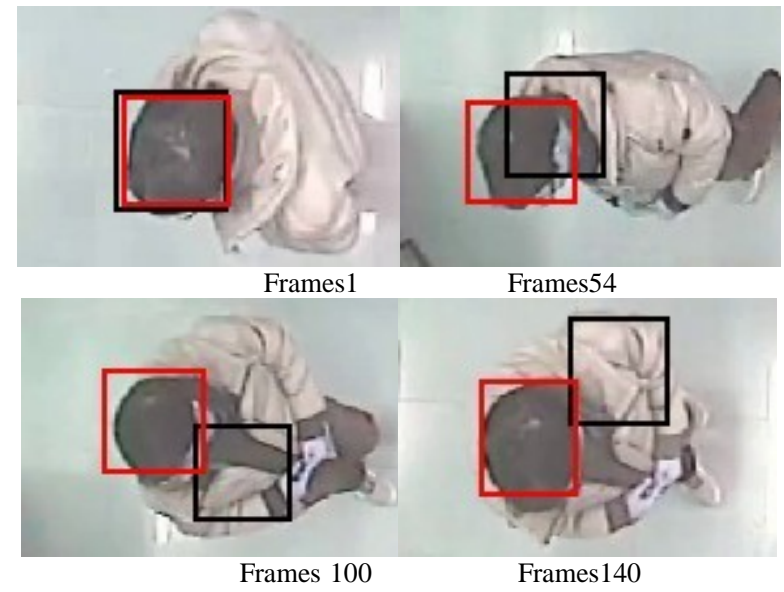

Figure4. The comparison between our algorithm and the original

The second experiment proved that our algorithm is robust for the rapid movement of object. Both choose the color-texture features. Select a video sequence of a pedestrian head tracking. The pedestrian moves fast and speed changes suddenly at the frames 100. Figure.4 shows the results and the black rectangle for tracking results without the Kalman filter, the red for our algorithm. It can be seen that the speed of pedestrain changes and become faster at the frames 100 , our algorithm shows the effective.

\section{CONCLUSIONS}

In this paper we propose an effective algorithm of object tracking which uses color-texture histogram features and applying it to the MS framework, then Kalman is used to predict the object's location of the next frame and the center of object in each frame as the initial iteration of MS. Experimental results indicate that the proposed MS with the Kalman filter performs much better than the original algorithm, especially in tracking objects that have similar color appearance to the background or the object moves fast.

\section{REFERENCES}

[1] D.Comaniciu,V.Ramesh and P.Meer. Kernel-Based ObjectTracking[J]. IEEE Trans. on Pattern Analysis and Machine Intellengece, vol.25(5), pp.564-577, 2003.

[2] Fukunaga F, Hostetler L D. The estimation of the gradient of a density function with application in pattern recongnition[J]. IEEE Transactions on information Theory, 1975, 21(1):32-40

[3] Kirsch A.An introduction to the mathematical theory of inverse problems[M].New York: Springer-Verlag, 1999.

[4] Guo wu. Yuan, Dan. Xu. A combination of texture and color of the moving object tracking algorithm[J]. Computer application and software, 2011.28(11)

[5] Guan Bin. Li, He Feng. Wu. an object tracking algorithm based on color texture histogram with the right to block average drift[J]. Jounal of Computer-Aided Design \& Computer Graphics, 2011.23 (12)

[6] Kennedy J, Eberhart R C. Particle swarm optimization[C]. Proceedings of the IEEE International Conference on Neural Networks. Piscataway,NJ,Perth: IEEE Server Center,1995:1942-1948.

[7] T.Ojala, M.Pietikäinen and T.Mäenpää. Multiresolution gray scale and rotation invariant texture analysis with local binary patterns. In PAMI, 2002

[8] Ojala T. Valkealahti K. Oja E. et al. Texture discrimination with multidimensional of signed gray level differences[J]. Pattern Recognition. 2001, 34(3):727-739

[9] Ning J, Zhang D, et al. Robust object tracking using joint color-texture histogram[J]. International Journal of Pattern Recognition and Artificial Intelligence, 2009, 23(7):1245-1263 